\title{
Rapidly Growing and Recurrent Pulmonary Venous Leiomyosarcoma Involving the Left Atrium: Detected by Echocardiography with its Fatal Consequences - A Case Report
}

\author{
SK BISWAS ${ }^{\mathrm{a}}$, HITOSHI HISHIDA $^{\mathrm{b}}$, MIYUKI KIMURA ${ }^{\mathrm{c}}$, KUNIHIKO SUGIMOTO $^{\mathrm{d}}$, MASATSUGU IWASE ${ }^{\mathrm{e}}$
}

Summary:

Leiomyosarcoma is a rare form of malignant tumour of smooth muscle cells and its course is very unpredictable. One 19-year-old male patient with rapidly growing pulmonary venous leiomyosarcoma was detected by echocardiography in the Fujita Health University Hospital, Japan. Initially he developed exertional dyspnoea, paroxysmal nocturnal cough and haemoptysis. Symptoms were worsening rapidly. Contrast CT revealed left atrial dilatation and a filling defect at the commencement of right upper pulmonary vein and left atrium. Echocardiography showed a large, bilobular, echogenic mass within the left atrium and obstructing the mitral valve annulus during diastole. For maintaining the haemodynamic balance emergency operation was performed. Within very short interval subsequent two operations were performed due to

Introduction:

Leiomyosarcoma is relatively uncommon and account for $7 \%$ of soft tissue sarcomas. They occur in adult and affect women more frequently than man. Most of them

a. Dr. Shankar Kumar Biswas, M.Phil, Senior Medical Officer, Center for Nuclear medicine \& Ultrasound, Barisal, Bangladesh

b. Prof. Hitoshi Hishida, MD, Professor, Division of Cardiology \& Department of Internal Medicine, Fujita Health University, Japan.

c. Dr. Miyuki Kimura, MD, Assistant Professor, Division of Cardiology \& Department of Internal Medicine, Fujita Health University, Japan.

d. Mr. Kunihiko Sugimoto, RDCS, Echocardiographer, Joint Research Laboratory of Clinical Medicine, Fujita Health University, Japan.

e. Prof. Masatsugu Iwase, MD, Professor, Division of Cardiology \& Department of Internal Medicine, Fujita Health University, Japan.

Address of Correspondence: Dr. Shankar Kumar Biswas, Senior Medical Officer. Center for Nuclear Medicine and Ultrasound, Barisal. P.O. Box: 43, Bangladesh. Tel: 0431-64014(off); 0431217-5808(res); Email: biswas 70@yahoo.com recurrent mass at the same site. After $1^{\text {st }}$ operation chemotherapy and after $3^{\text {rd }}$ operation radiotherapy were given but the patient developed metastatic lesions in brain and abdomen. He subsequently underwent operation for focal brain lesion. The histological and immunohistochemical appearance was that of an intimal sarcoma, most closely resembling malignant histiocytoma. Usually $1^{\text {st }}$ operation is possible in primary leiomyosarcoma and due to rapid metastasis subsequent operations are not possible, but this case was an exceptional one. Enbloc dissection was carried out in three stages, each time histological diagnosis was debatable and finally confirmed as primary pulmonary venous leiomyosarcoma on autopsy specimen.

Key words: Leiomyosarcoma, Echocardiography, Immunohistochemistry.

(J Bangladesh Coll Phys Surg 2006; 24 : 122-126)

develop in the skin and deep soft tissues of the extremities and retroperitoneum. Superficial or cutaneous lesions are usually small and have a good prognosis, whereas those are large, can't be entirely excised, cause death by both local extension and metastatic spread ${ }^{1}$. Here we report a case that was detected in the Fujita Health University Hospital in 1998 and operated thrice within 4 months to alleviate haemodynamic disturbances. But due to rapid progression and metastasis to abdomen and brain the patient expired of about 13 months of initial diagnosis.

\section{Case Report:}

A 19 year old male patient developed dyspnoea on exertion, paroxysmal nocturnal dyspnoea and haemoptysis; on chest X-ray no abnormality could be detected. Recurrent cough was initially treated as bronchitis and his symptoms were slightly improved. Due to low grade fever, worsening haemoptysis and dyspnoea he was admitted in the local hospital, where chest X-ray and CT scan without enhancement revealed dilatation of upper pulmonary vessels, alveolar type of pulmonary oedema and bilateral pleural effusion. A 
bronchofiberscopy confirmed the dilated bronchial tree and oedema of the alveolar membrane but no evidence of malignancy was noted. All investigations prompted towards heart failure and pulmonary hypertension. Further workup with 2D echocardiography showed a mass in the left atrium and then he was referred to the Fujita Health University Hospital for proper management. On admission ECG revealed tachycardia, left axis deviation, broad and notched $\mathrm{p}$ wave in the lead II, III and aVF; chest X-Ray revealed pneumonic consolidation, pulmonary congestion, dilated pulmonary trunk without evidence of cardiomegaly. 2D echocardiography showed a large, bilobular, echogenic mass invading the left atrium, prolapsed across the mitral valve into the left ventricle during diastole and obstructing the mitral valve annulus.

In transoesophageal echocardiography, left atrium was filled with so large tumour as to obstruct right upper pulmonary venous outflow. This represented as the increased peak velocity of the flow by pulse wave Doppler echocardiography (Fig.-1). Contrast CT revealed left atrial dilatation and a filling defect at the commencement of right upper pulmonary vein and left atrium (Fig.-2). The maximum velocity was increased as to $350 \mathrm{~cm} / \mathrm{sec}$ by continuous wave Doppler

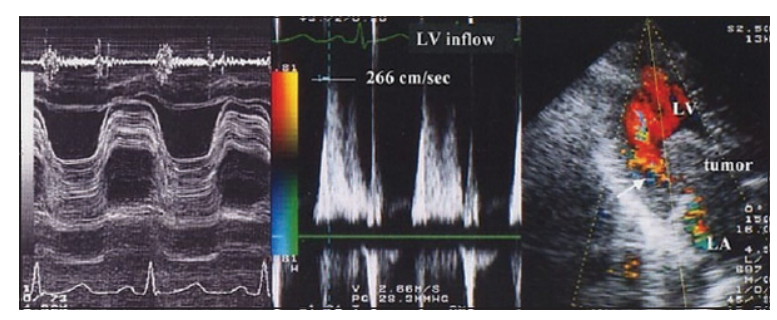

Fig.-1: Doppler echocardiography shows intracardiac mass \& increased peak velocity $(266 \mathrm{~cm} / \mathrm{sec})$ through the mitral valve.
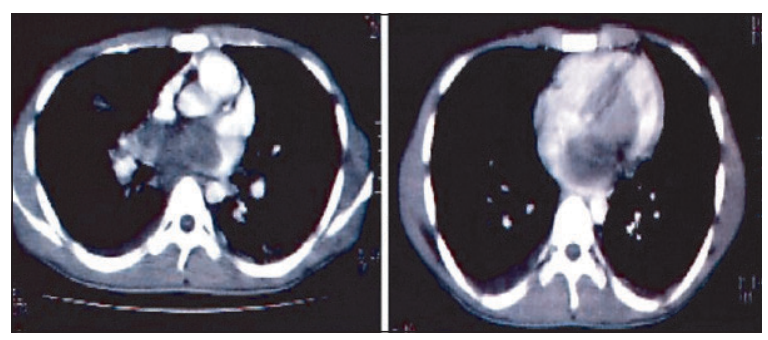

Fig.-2: Contrast CT shows hypodense mass within the left atrium involving the root of the pulmonary veins. echocardiography; as a result pulmonary venous pressure also raised. Severe tricuspid regurgitation was noted with a peak velocity was $400 \mathrm{~cm} / \mathrm{sec}$ and the maximal right ventricular pressure was $74 \mathrm{mmHg}$ calculated by modified Bernoulli equation. Due to pulmonary oedema and pulmonary hypertension, arterial blood gas analysis showed hypoxemia $\left(\mathrm{SO}_{2}=95.9 \%\right.$ and $\mathrm{PO}_{2}=68.7 \mathrm{mmHg}$ ) with oxygen inhalation of $4 \mathrm{~L} /$ minute through nasal canula. To relieve cardiopulmonary insufficiency operative decision was taken at the day after admission in an emergency basis and preoperatively it was thought to be cardiac tumour. The tumour was excised with total cardiopulmonary bypass support under moderate hypothermia $\left(34^{\circ} \mathrm{C}\right)$ with bicaval cannulation for venous drainage. After an incision in right atrium, interatrial septum was incised vertically through fossa ovalis. The tumour was about $100 \mathrm{gm}$, arose from right upper pulmonary vein and extended into the left atrium, filling the lumen of the right upper pulmonary vein but the occlusion was easily relieved. The tumour also attached to the anterior free wall of the left atrium. The two regions of attachment of the tumour were carefully excised and the tumour was removed enbloc (Fig.-4). The resultant atrial septal defect was closed with a knitted Dacron patch. The atrial chambers and atrioventricular valve areas were carefully searched for additional tumour implants before closer of atrial incision. The surface of the resected specimen was smooth, mimicking a benign mass.

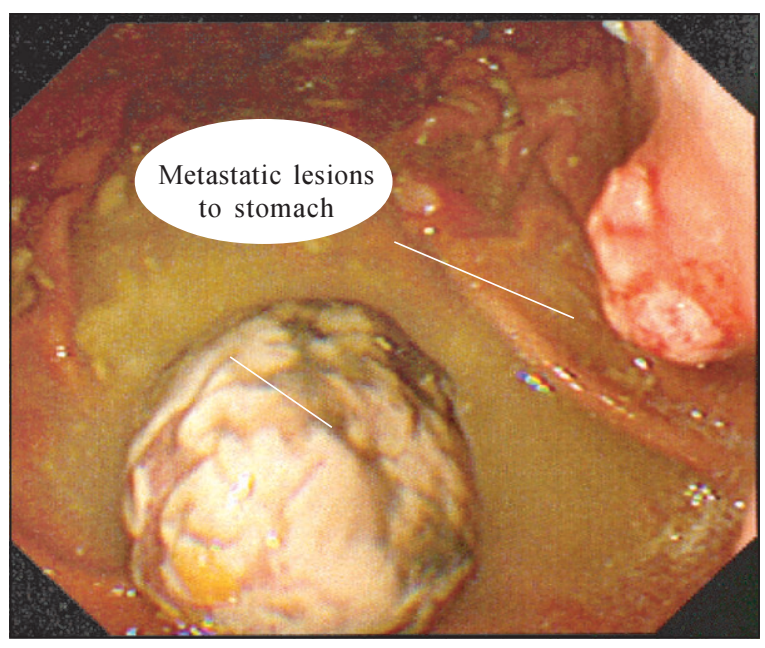

Fig.-3: Endoscopic view of the metastatic lesions in the stomach. 


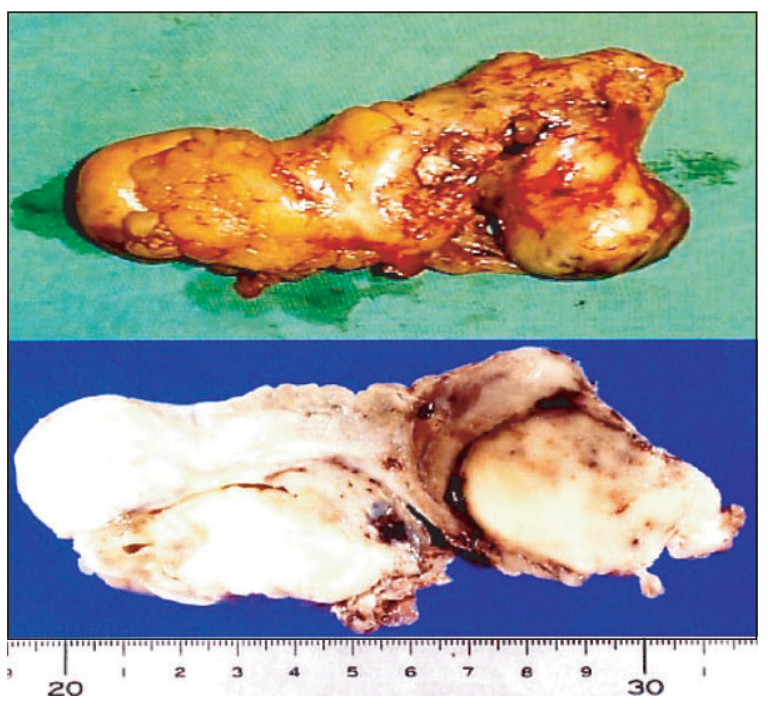

Fig.-4: Morphological appearance of the tumour.

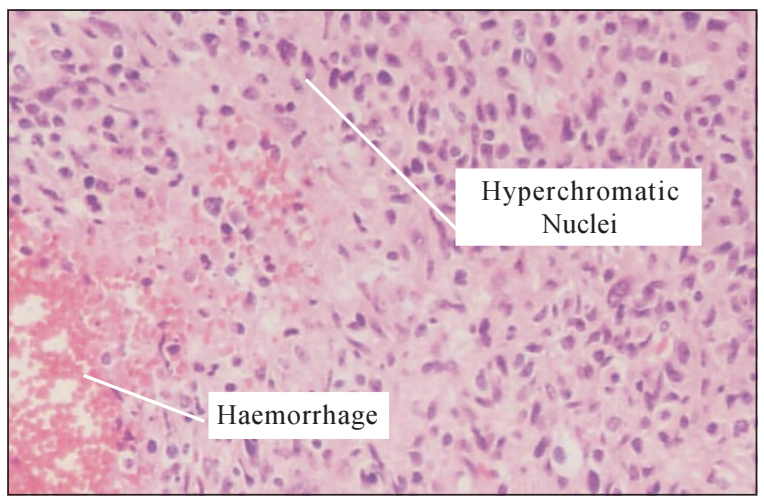

Fig.-5: Histopathological appearance of the tumour showing hyperchromatic nuclei \& haemorrhage.

Histologically confirmed that the tumour was primary sarcoma and after resection the patient had symptomatically improved. Though complete excision was expected but it could not possible because sarcomas were microscopically present at the excised margin of the organ involved i.e. in the intima of randomly selected 3 sites- in the left atrium, interatrial septum and right upper pulmonary vein. He received postoperative adjuvant chemotherapy based on fluorouracil as an ambulatory care. But on $26^{\text {th }}$ day of resection an echogenic mass of about $10 \mathrm{~mm}$ in diameter, appeared at the boundary of the right upper pulmonary vein and left atrium, detected by transoesophageal echocardiography. It was thought to be recurrence and after 35 days (after 41 days of first operation) of detection of recurrent mass he suddenly developed haemoptysis and dyspnoea; chest X-ray revealed pulmonary congestion. In $2 \mathrm{D}$ echocardiography the recurrent mass was appeared to be prolapsing and obstructing the mitral valvular annulus. The maximal right ventricular pressure was more than $62 \mathrm{mmHg}$, calculated by continuous wave Doppler echocardiography. His symptoms were thought to be due to pulmonary oedema and pulmonary hypertension. To relieve pulmonary hypertension $2^{\text {nd }}$ operation was performed as an emergency basis. The recurrent mass was about 100 $\mathrm{gm}$, also arose from the right upper pulmonary vein and it was carefully excised enbloc with the venous wall. Histologically this sarcoma was considered to be leiomyosarcoma arising from the right upper pulmonary vein.

After $2^{\text {nd }}$ operation the patient was symptomatically improved and there was no circulatory abnormality but after about 3 months he developed almost same sized recurrent mass at that particular site, detected by follow up echocardiography and accordingly operation was performed. After $3^{\text {rd }}$ operation radiotherapy was applied to prevent recurrence.

On follow up echocardiography after 6 months of $3^{\text {rd }}$ operation no recurrence was revealed but the patient developed huge abdominal swelling due to ascites. Ultrasound, endoscopy, contrast CT and MRI revealed that there was a big soft tissue mass in the pelvic cavity, metastasis to stomach (Figure-3) and enlarged para aortic lymph nodes, but no focal lesion was detected in liver. Operation was not performed for the pelvic mass and after few days the patient developed features of space occupying lesion in the Brain. Contrast CT and MRI confirmed about metastatic lesion in the Brain. The patient underwent brain operation and histopathology of the specimen showed the same features of cardiac tumour; that showed spindle shaped irregularly arranged different sized hyperchromatic nuclei, increased mitotic activity with evidence of hemorrhage and necrosis (Fig.5). He died after 3 months due to disseminated abdominal tumours though there was no recurrence of cardiac or pulmonary venous tumour.

\section{Discussion:}

Cardiac leiomyosarcoma is a very rare entity that is found in less than $0.2 \%$ of all cardiac tumours. At the time of primary diagnosis, it often shows advanced local 
invasion or may even be metastasized. Thus complete resection can not easily be achieved. Though cardiac transplantation has been reported as a therapeutic option but radical redo-extirpation was done in a case of cardiac leiomyosarcoma without the necessity of transplantation ${ }^{2}$.

The natural history of leiomyosarcoma is very unpredictable. It is not very responsive to chemotherapy or radiotherapy. The best outcome occurs when surgical excision with wide margins are done. Staging has an important role in determining the most effective treatment of soft tissue sarcoma. While low grade tumours are usually curable by surgery alone, higher grade sarcomas are associated with higher local treatment failure rates and increased metastatic potential.

This patient had histological confirmation of primary sarcoma; it invaded the innermost media focally and extended along small tributaries. Immunohistochemical examination was performed on formalin fixed, paraffinembeded sections by an indirect method. The tumour cells were positive for vimentin, alpha smooth muscle actin and human smooth muscle actin. They were negative for cytokeratins, desmin, Q Bend / CD34, S100 protein. There was no dysplasia in the adjacent endothelium. The appearance was those of an intimal sarcoma most closely resembling malignant histiocytoma but autopsy confirmed about leiomyosarcoma. In a case of primary leiomyosarcoma Goto et al observed that histological examination revealed spindle cell sarcoma with an interlacing pattern, acidophilic cytoplasm and blunt ended or 'Cigar-shaped nuclei'; immunohistochemical examination showed positive staining for vimentin, desmin and alpha smooth muscle actin ${ }^{3}$.

According to Ridwelski et al, for a primary leiomyosarcoma of blood vessel, a thorough diagnostic assessment includes sonography, computed tomography, angiography or duplex ultrasonography, perioperative pathological examination and appropriate differential diagnosis ${ }^{4}$. Baldwin reported a sarcoma arising from the left atrium in 1910, after that there were a few reports of sarcoma arising from pulmonary vein $5,6,7,8,9,10$. In the all previous reports but exception for one, the tumour had not defined in the histological classification. Keiser and Urmacher reported malignant histocytoma involving the pulmonary vein by resection ${ }^{6}$.
Tumour in the pulmonary artery was more likely myxoid than those in the aorta and has a propensity for osteoid formation, areas resembling malignant histiocytoma. Those in aorta more likely showing surface layering with an undersurface of dense collagenized tissue ${ }^{11}$. Those in vein have similar microscopic appearance to tumours of arterial origin 6 . In this case tumour revealed rapidly growing and filled the left atrium within 35 days only. The growth was so rapid that there was no way to overcome circulatory failure other than resection of the tumour thrice. It is very unusual because usually $1^{\text {st }}$ operation is possible in leiomyosarcoma but subsequent operation is not possible due to rapid metastasizing nature of the tumour. There are no previous reports of sarcoma to best of our knowledge that document the rapidity of its growth in vivo. Noncardiac sarcomas have demonstrated that histologic grading correlates with survival $^{12}$. Due to demonstration of mutations in some cases of leiomyosarcoma, the possibility of multifocal sarcoma should be kept in mind ${ }^{13}$. Burke et al reported that the mitotic activity and necrosis were considered to be the factors of poor prognosis. Primary sarcoma of either pulmonary artery or vein result in a dismal outcome with the diagnosis usually made at autopsy reflecting the poor prognosis. So the aim of resection may be to relieve symptoms of intracardiac obstruction or tamponade ${ }^{14}$.

Most of the primary cardiac tumours are highly aggressive; the average time of survival had been six months from the time of diagnosis ${ }^{15}$. So the treatment of cardiac sarcoma had been controversial with some authors, recommending radiation therapy and others preferring complete resection ${ }^{16}$. Recently increased clinical awareness and advanced diagnostic techniques have lead to more accurate diagnosis and early resection. There are some reports of longer time of survival but there was only one case survived more than 5 years after radiotherapy with radiosensitizer ${ }^{17}$.

Aggressive and complete surgical resection remains the only hope for palliation and survival in cardiac sarcoma and fatal rare tumours ${ }^{18,19}$. Murphy et al reported 5 cases, survives from 14 months to 3.5 years after complete resection ${ }^{19}$. Post operative chemotherapy didn't enhance survival of patients with incomplete resection. There is reasonable hope that leiomyosarcoma of the inferior vena cava can be treated successfully, 
even in advanced stages with novel antineoplastic drugs and radiotherapeutic protocols, however general treatment recommendations have not yet been compiled $^{19}$.

\section{Conclusion:}

Primary leiomyosarcoma detected by echocardiography was rapidly growing and due to its recurrence the patient underwent operation thrice at an interval of 1 and 3 months. Along with that chemotherapy and radiotherapy were used though that kind of adjuvant did not enhance longevity. Metastasis of tumour to pelvic cavity, stomach and brain were noted in absence of recurrence at the primary site. Usually $1^{\text {st }}$ operation is possible in primary leiomyosarcoma and due to rapid metastasis subsequent operation is impossible, but this case was exceptional. The tumour was not very invasive so enbloc dissection was possible in every attempt of operation and autopsy confirmed primary pulmonary venous leiomyosarcoma.

\section{Acknowledgement:}

My deepest gratitude and gratefulness are extended to all members of the Japan Society of Ultrasonics in Medicine (JSUM) and Bangladesh Society of Ultrasonography (BSU).

\section{References:}

1. Andrew ER. Skeletal system and soft tissue tumours. In: Cotran RS, Kumar V, Robbins SL eds. Robbins Pathologic basis of disease, $5^{\text {th }}$ ed, W.B.Saunders Company, 1994: pp 1268

2. Wippermann J, Albers J M, Brandes H, Wahlers T. RedoExtirpation of a cardiac Leiomyosarcoma to avoid Transplantation.Thorac Cardiovasc Surg 2002; 50: 62-3.

3. Goto $\mathrm{T}$, Ishida $\mathrm{T}$, Motoi $\mathrm{N}$ et al. Primary Leiomyosarcoma of the Femur. Journal of Orthopaedic Science 2002; $7(2): 267-73$.

4. Ridwelski K, Rudolph S, Meyer F et al. Primary Sarcoma of the inferior vena cava: review of diagnosis, treatment \& outcome in a case series. International Surgery $\quad 2001 ; 86$ (3):184-9

5. Baldwin HA. Primary sarcoma of the heart, with report of additional case. JAMA 1910; 55: 646 .

6. Kaiser LR, Urmacher C. Primary sarcoma of the superior pulmonary vein.Cancer $\quad 1990 ; 66: 789-95$.

7. Ravid JM, Sachs J.Tumours of the heart. Am Heart J 1943; 26:385-97.

8. Coming ARR, Shillitoe AJ. Ball valve mitral obstruction by a sarcoma of pulmonary vein. Br Heart $J$ 1957; 19:287-9.

9. Kidd BSL, Carson DJL, Lamont ES. Intra-atrial sarcoma. $\mathrm{Br}$ Med $J$ 1961; 2:1476-78.

10. Haber LM, Troung L. Immunohistochemical demonstration of the endothelial nature of aortic intimal sarcoma. Am J Surg Pathol 1988; 12:798-802.

11. Burke AP, Vitmani R. Sarcomas of the great vessels: a clinicopathologic study. Cancer 1993; 71:1761-73.

12. Ueda T, Aozasa K, Tsujimoto M et al. Multivariate analysis for clinical prognostic factors in 163 patients with soft tissue sarcoma. Cancer 1988; 62:1444-50.

13. Bohm P, Tieben C, Pressler H. Multifocal soft tissue metastasis of gastric leiomyosarcoma. Archives of Orthopaedic \& trauma surgery 2002; 122(2): 112-4.

14. Burke AP, Cowan D, Virmain R. Primary sarcomas of the heart. Cancer 1992; 69:387-95.

15. Dein JR, First WH, Stinson EB. Primary Cardiac neoplasms: early and late results of surgical treatment in 42 patients.J Thorac cardiovasc Surg 1987; 93: 511-20.

16. Poole GC J, Meredith JW, Breyer RH, Mills SA. Surgical implications in malignant cardiac disease. Am Thorac Surg 1983; 36:484-91

17. Movsas B, Teruya-Feldstein J, Smith J, Glatstein E, Epstein AH. Primary cardiac sarcoma 1998; 114:648-52.

18. Putnam JB J, Dweeney MS, Colon R, Lanza LA, Frazier $\mathrm{OH}$, Cooley DA. Primary cardiac sarcomas. Am Thorac Surg 1991; 51: 960-70

19. Murphy MC, Sweeney MS et al. Surgical treatment of Cardiac tumours: a 25 yrs experience. Am Thorac Surg 1990; $49: 612-8$ 